Canadian

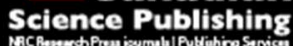

Applied Physiology, Nutrition, and Metabolism Physiologie appliquée, nutrition et métabolisme

\title{
A pilot study investigating reactive oxygen species production in capillary blood after a marathon and the influence of an antioxidant-rich beetroot juice
}

\begin{tabular}{|r|l|}
\hline Journal: & Applied Physiology, Nutrition, and Metabolism \\
\hline Manuscript ID & apnm-2017-0587.R1 \\
\hline Manuscript Type: & Brief communication \\
\hline Complete List of Authors: & $\begin{array}{l}\text { Clifford, Tom; Newcastle University } \\
\text { Bowman, Amy; Newcastle University } \\
\text { Capper, Tess; Newcastle University } \\
\text { Allerton, Dean; Newcastle University } \\
\text { Foster, Emma; Newcastle University } \\
\text { Birch-Machin, Mark; Newcastle University } \\
\text { Lietz, Georg; Newcastle University } \\
\text { Howatson, Glyn; Northumbria University, Department of Sport Health and } \\
\text { Rehabilitation } \\
\text { Stevenson, Emma; Newcastle University }\end{array}$ \\
\hline Is the invited manuscript for \\
consideration in a Special \\
Issue? :
\end{tabular}


1 A pilot study investigating reactive oxygen species production in capillary 2 blood after a marathon and the influence of an antioxidant-rich beetroot juice 3

4 Tom Clifford ${ }^{1}$, Amy Bowman², Tess Capper², Dean M. Allerton ${ }^{1}$, Emma Foster ${ }^{2}$, 5 Mark Birch-Machin ${ }^{2}$, Georg Lietz ${ }^{2}$, Glyn Howatson ${ }^{3,4}$, Emma J. Stevenson ${ }^{2}$

$6 \quad{ }^{1}$ School of Biomedical Sciences, Newcastle University, United Kingdom; ${ }^{2}$ Institute of 7 Cellular Medicine, Newcastle University, Newcastle, United Kingdom; ${ }^{3}$ Department of 8 Sport, Exercise and Rehabilitation, Northumbria University; ${ }^{4}$ Water Research Group,

9 School of Environmental Sciences and Development, Northwest University,

10 Potchefstroom, South Africa. Emails: tom.clifford@ncl.ac.uk;

11 amy.bowman@ncl.ac.uk; t.capper2@ncl.ac.uk; dean.allerton@ncl.ac.uk;

12 emma.foster@ncl.ac.uk; mark.birch-machin@ncl.ac.uk; Georg.lietz@ncl.ac.uk;

13 glyn.howatson@northumbria.ac.uk; emma.stevenson@ncl.ac.uk.

Address for correspondence:

16 Tom Clifford

17 Newcastle University

18 Faculty of Medical Sciences

19 School of Biomedicine

20 Newcastle-upon-Tyne

21 NE2 4HH

22 UK

23 Tel: +440192088311 


\section{$30 \quad$ Abstract}

31 We report that reactive oxygen species (ROS), as measured in capillary blood taken 32 from the finger-tip, increased after a marathon $(+128 \% P<0.01$; $E S=1.17)$, 33 indicating that this collection method might be useful for measuring ROS in field 34 settings. However, mitochondrial DNA damage remained unchanged. Beetroot juice, 35 taken before and after exercise, was unable to mitigate exercise induced-ROS 36 production, questioning its use an antioxidant rich-food.

37

38 Key words: Reactive oxygen species; running; beetroot; exercise

39

40

41

42

43

44

45

46

47

48

49

50

51

52

53

54

55

56

57

58 
59 Introduction

60 To measure exercise-induced ROS generation rand the effectiveness of antioxidant 61 (AOX) supplements, a number of biomarkers have been developed. These 62 biomarkers are most commonly measured in blood matrices, given that muscle 63 tissue can only be obtained with highly invasive biopsy procedures (Nikolaidis et al., 64 2012). Collecting venous blood samples can also be problematic, however; as well 65 as being time consuming, it requires specialist expertise, meaning that monitoring 66 ROS outside of the lab, in clinics or applied sport settings for instance, is very limited 67 (Twist and Highton, 2013). One potential solution to this problem is the use of less 68 invasive techniques. In this regard, we have developed methods that estimate 69 physiological stress from a finger-tip capillary blood sample that measures either 70 ROS production or damage to mitochondrial DNA (mtDNA). Collecting blood from 71 the finger-tip is quick, minimally invasive, and requires little equipment, and therefore 72 offers several advantages in applied or clinical point of care research settings. Thus, 73 one of the aims of this study was to investigate the suitability of these measures for 74 estimating physiological stress following strenuous exercise.

75 Another aim of this study was to assess the effectiveness of an AOX-rich food 76 (beetroot juice; BTJ) for attenuating the ROS production. Numerous studies have 77 shown that the constituents in beetroot, chiefly the betalain pigments, are potent 78 AOX that have the potential to attenuate ROS generation (Clifford et al., 2015). 79 However, most of these observations have been carried out either in vitro or in 80 animal studies so these findings might not be directly transferable to the in vivo 81 environment in humans. Accordingly, in this pilot study we wanted to; 1) to examine 82 the effects of a ROS production and mtDNA damage in capillary blood after a 83 marathon and; 2) to establish whether post-exercise these markers can be 84 attenuated with BTJ. We selected a marathon because the high physiological stress 85 imposed has been shown to serve as a good model to provoke a robust increase in 86 ROS production and inflammatory related events (Gomez-Cabrera et al., 2006; 87 Nieman et al., 2002).

88

\section{$89 \quad$ Methods}

90 Participants 
91 Fourteen, healthy marathon runners volunteered to participate in this study. A 92 summary of their physical characteristics and training history are presented in Table

93 1. The protocol received ethical approval from Newcastle University (16LIE016) and 94 all participants provided informed consent.

95 Experimental design

96 This study was a double blind, placebo-controlled, independent group's trial with two 97 experimental treatment arms: BTJ $(n=7)$ or a placebo (PLA) $(n=7)$. Capillary 98 puncture samples from the finger-tip were obtained pre-supplementation, pre-race, 9930 mins post-race and the morning following the race. Online dietary recall software 100 (Intake24, Newcastle University, UK) was used to record participant's dietary intake 101 the day before and on the day of the race.

102 Supplementation

103 The BTJ used in this study was Love Beets Beetroot Juice (Gs Fresh Ltd, 104 Cambridgeshire, UK) and the PLA used in this study was water mixed with 105 maltodextrin (Myprotein, Manchester, UK) and a fruit squash (Kia Ora, Coca Cola, 106 UK) to match the BTJ for carbohydrate and energy content. Details on the 107 polyphenol and AOX content of these two drinks - as well, as how they compare to 108 other antioxidant-rich beverages, can be found in Clifford et al., (2016). As in a 109 similarly designed, study (Howatson et al., 2010), participants consumed their 110 respective supplements twice daily (250 $\mathrm{ml}$ per serving) at 08:00 and 20:00 on the 4 111 days prior to the marathon, on the day of the marathon and at 08:00 the morning 112 after the marathon (11 servings in total). To ensure the drinks were provided in a 113 double-blind fashion, both supplements were prepared in masked bottles that were 114 identical in size and appearance.

\section{Blood sampling and analysis}

116 Capillary blood samples were analysed for haemoglobin $(\mathrm{Hb})$ concentrations 117 (Hemocue 201+; HemoCue AB, Angelholm, Sweden), ROS production, and mtDNA 118 damage. ROS production was measured in whole blood using a luminescence assay 119 according to the methods described by Yamazaki et al., (2011). $2 \mu$ l of blood was 120 immediately added to a buffer at a dilution of 1:50 blood to buffer. Phorbol myristate 121 acetate (PMA) was later added to whole blood to stimulate ROS production in blood 122 leukocytes. The pre and post-race samples were kept on ice until transported by car 
123 to the University, where they were analysed within $24 \mathrm{~h}$ of race completion. The 124 assay established by PB Biosciences Ltd (Newcastle, UK) is based on the principle 125 that luminol is oxidised by ROS to produce light, which is measured over 22 minutes 126 in real time, and the area under the curve determined. The inter-assay coefficient of 127 variation (CV) for this technique was established as $9.9 \%$. It is important to 128 acknowledge that because this assay is performed at an ambient $\mathrm{PO}_{2}, \mathrm{ROS}$ 129 production might be overestimated compared to in vivo where $\mathrm{PO}_{2}$ is lower.

130 MtDNA damage was measured via real-time quantitative PCR (qPCR). DNA was 131 extracted from a blood spot on an FTA card (GE Healthcare Life Sciences), using a 132 QIAamp DNA Mini Kit (Qiagen, UK) with the manufacturer's protocol for extracting 133 DNA from dried blood spots modified. Six punched-out circles from a dried blood 134 spot were placed in a $1.5 \mathrm{ml}$ microcentrifuge tube, for each time point of each 135 volunteer. To these tubes, $190 \mu$ l Buffer ATL was added and the tubes incubated at $13685^{\circ} \mathrm{C}$ for 10 minutes. Following incubation, $10 \mu$ proteinase $\mathrm{K}$ was added and 137 samples were incubated at $56^{\circ} \mathrm{C}$ for 1 hour, with pulse-vortexing every 15 minutes 138 for 15 seconds. Samples were incubated at $95^{\circ} \mathrm{C}$ for 5 minutes, $200 \mu \mathrm{l} \mathrm{Buffer} \mathrm{AL}$ 139 added, and samples incubated at $70^{\circ} \mathrm{C}$ for 10 minutes. Extraction was then 140 continued according to the manufacturer's protocol (Qiagen, UK), and samples were 141 stored at $4^{\circ} \mathrm{C}$ until analysis. DNA concentrations were determined using an ND-1000 142 Nanodrop Spectrophotometer (Thermo Scientific, UK) at a wavelength of $260 \mathrm{~nm}$. To 143 perform the qPCR reaction, the following components were assembled on ice to a 144 final volume of $20 \mu \mathrm{l}$ per well of a MicroAmp Fast Optical 96-Well Reaction Plate 145 (Applied Biosystems, UK): dH20, 1x SensiMix SYBR Hi-ROX (Bioline, UK), $0.25 \mu \mathrm{M}$ 146 each of the forward primer (AL4_F, CTGTTCTTTCATGGGGAAGC) and the reverse 147 primer (AS1_R AAAGTGCATACCGCCAAAAG) (Eurofins MWG Operons, 148 Germany), and 12 ng DNA. QPCR was performed using a StepOnePlus Real-Time 149 PCR System (Applied Biosystems, UK) with the results viewed using StepOne 150 Software V2.1 (Applied Biosystems, UK). The following conditions were used for the 151 qPCR run: $95^{\circ} \mathrm{C}$ for 10 minutes; 40 cycles of $95^{\circ} \mathrm{C}$ for 15 seconds, $60^{\circ} \mathrm{C}$ for 15 152 seconds, and $72^{\circ} \mathrm{C}$ for 55 seconds; and a final stage of $72^{\circ} \mathrm{C}$ for 7 minutes. A melt 153 curve was added immediately after the reaction with the conditions of: $95^{\circ} \mathrm{C}$ for 15 154 seconds; $60^{\circ} \mathrm{C}$ for 1 minute, followed by a plate read at every $0.3^{\circ} \mathrm{C}$ temperature 155 increase; ending at $95^{\circ} \mathrm{C}$ for 15 seconds. The $\mathrm{CV}$ for this technique is $<1 \%$. 
156 Data Analysis

157 All data are expressed as mean \pm SD and statistical significance was set at $P<0.05$.

158 Differences in participant group characteristics, training history and dietary intake 159 were analysed with multiple student t-tests. Prior to analysis, ROS production was 160 adjusted for changes in plasma volume using the methods of Dill and Costill (1974). 161 A mixed model ANOVA with 2 independent group levels (BTJ vs. PLA) and 4 162 repeated measures time points (pre-supplementation, pre-race, post-race and day 1 163 post) was used to analyse for group differences in ROS production and mtDNA 164 damage. Bonferroni post hoc analysis was performed to locate any significant 165 differences. Data were analysed using GraphPad (GraphPad, Prism, CA, US).

\section{Results}

167 There were no differences in physical characteristics, training history and marathon 168 performance between the two groups (see Table $1 ; P>0.05$ ), and no differences in 169 average dietary intake the day before and on the day of the marathon 170 (supplementary table S1). ROS was unchanged from pre-supplementation to pre171 race, irrespective of supplement $(P>0.05$; $\mathrm{ES}=1.96)$. Immediately after the 172 marathon ROS production increased by $128 \%$ (average across groups; time effect; $P$ $173<0.01$; ES = 1.17; Figure 1), but returned to pre-exercise levels the following day 174 (ES = 0.57). At no time point did the level of ROS differ between the BTJ and PLA 175 groups $(P>0.05)$. The marathon did not induce mtDNA damage (pre to post176 marathon change; $P>0.05$, ES $=0.36$ ) and did not differ between BTJ and PLA 177 post-marathon $(E S=0.51)($ Figure 2).

\section{Discussion}

179 Systemic ROS production in whole blood samples was increased immediately after 180 the marathon before returning to resting levels the following morning. These findings 181 appear to be consistent with others (Gomez-Cabrera et al., 2006; Nieman et al., 182 2002), who employed indirect markers to measure ROS generation after long 183 distance running (malondialdehyde and lipid hydroperoxides, $\mathrm{F}_{2^{-}}$isoprostanes, 184 respectively). The clear advantage of this method for detecting ROS generation over 185 those used in previous studies is that only a very small amount of blood is required 186 from the finger-tip $(2 \mu \mathrm{l})$. Therefore, it can be useful for estimating ROS in settings 187 outside of the laboratory, when other methods of blood collection (i.e., venepuncture) 188 might be time consuming and impractical. Another advantage of this method is that it 
189 gives a direct indication of radical production in the blood, as opposed to an 190 estimation based on (presumably) radical-mediated damage to molecules such as 191 proteins, fats or DNA as other point of care methods tend to do. Future research 192 testing the agreement of this method with the most current valid and reliable 193 methods of exercise-induced ROS detection is warranted.

194 Interestingly, although not statistically significant, the ES for an increase in ROS 195 production from pre-supplementation to pre-race was very large. We are not clear 196 why this was the case, but it was evident in both groups, suggesting it was not 197 related to the drinks. One possible explanation is that pre-race nerves imposed a 198 degree of psychological stress sufficient to elevate oxidative stress above baseline 199 levels. Such a possibility should be examined in future work.

200 To the best of our knowledge, the current study is the first study to measure mtDNA 201 deletion in blood after endurance exercise, and use micro-invasive techniques; 202 nevertheless, akin to the findings of Beiter et al., (2011), who measured mtDNA 203 damage after an exhaustive treadmill run, we did not find any evidence of circulatory 204 mtDNA damage immediately or the morning after a marathon. The exact source of 205 circulating mtDNA after exercise is unclear (Nasi et al., 2016), but release from 206 muscle is probable (Nasi et al., 2016). In this case, it is possible that ROS induced 207 by the marathon was not sufficient to induce a large efflux of mtDNA into the 208 circulation, and perhaps this could explain why we were unable to detect changes 209 from pre to post-exercise.

210 BTJ was no more effective than a PLA for attenuating the rise in ROS generation or 211 mtDNA damage after a marathon. Our findings are in contrast to the work in animals, 212 which has consistently shown that BTJ, beetroot extracts, or its pigments, the 213 betalains, reduce ROS generation in response to a xenobiotic challenge (Clifford et 214 al., 2015). It is not clear exactly why our findings are inconsistent with others, but 215 differences in species and participants, the type and dose of beetroot administered, 216 oxidant stimulus, and ROS markers used-along with their method of assessment217 could all provide plausible explanations. Yet, another possibility is that we were 218 simply underpowered to detect small changes in ROS generation between the BTJ 219 and the PLA groups, given the low number of participants per group $(n=7)$. Indeed, to 220 observe a $10 \%$ difference in our primary outcome measure, ROS production, it is 221 estimated that we would need 16 participants per group (at $80 \%$ power and $\alpha$ of 
222 0.05). We acknowledge that this is a key limitation of the study and stress that this 223 was only a pilot study and, thus, these findings should be treated as preliminary.

224 In conclusion, we have shown that measuring ROS production in only $2 \mu$ of blood 225 from a finger-tip holds potential as a minimally invasive, quick, and simple method of 226 detecting ROS generation in applied sport settings outside of the laboratory. In 227 contrast, circulatory mtDNA damage was unaffected by a marathon. Finally, BTJ did 228 not mitigate ROS generation after the marathon and, thus, its benefits as an 229 exogenous antioxidant in humans remain questionable. Further research with larger 230 samples sizes are warranted to clarify these findings.

231

\section{Conflict of interest}

233 This study was funded as part of a doctoral degree that receives financial support 234 from Gs Fresh Ltd. The funders supplied the supplements used in this study but had 235 no role in the conception of the study, its design, preparation, analysis and writing of 236 the manuscript. The authors declare no conflict of interest.

\section{Reference list}

239 Beiter, T., Fragasso, A., Hudemann, J., Nieß, A. M., \& Simon, P. 2011. Short-term 240 treadmill running as a model for studying cell-free DNA kinetics in vivo. Clin. Chem. 241 57(4): 633-636. DOI: 10.1373/clinchem.2010.158030. PMID: 21296972.

242 Clifford, T., Howatson, G., West, D.J. and Stevenson, E.J., 2015. The potential 243 benefits of red beetroot supplementation in health and disease. Nutrients 7(4):2801244 2822. DOI: 10.3390/nu7042801. PMID: 25875121.

245 Clifford, T., Constantinou, C. M., Keane, K. M., West, D. J., Howatson, G., \& 246 Stevenson, E. J. 2016. The plasma bioavailability of nitrate and betanin from Beta 247 vulgaris rubra in humans. Eur J Nutr. 56(3): 1245-1254. DOI: 10.1007/s00394-016248 1173-5. PMID: 26873098. 
249 Dill, D. B., \& Costill, D. L. 1974. Calculation of percentage changes in volumes of 250 blood, plasma, and red cells in dehydration. J Appl Physiol. 37(2): 247-248. PMID: 2514850854.

252 Gomez-Cabrera, M. C., Martínez, A., Santangelo, G., Pallardó, F. V., Sastre, J., \& 253 Vina, J. 2006. Oxidative stress in marathon runners: interest of antioxidant 254 supplementation. Brit J Nutr. 96(S1): S31-S33. PMID: 16923247.

255 Howatson, G., McHugh, M. P., Hill, J. A., Brouner, J., Jewell, A. P., van Someren, K., 256 et al. 2010. Influence of tart cherry juice on indices of recovery following marathon 257 running. Scand J Med Sci Sports. 20(6): 843-852. DOI: 10.1111/j.1600258 0838.2009.01005.x. PMID: 19883392.

259 Nasi, M., Cristani, A., Pinti, M., Lamberti, I., Gibellini, L., De Biasi, S., et al. 2016. 260 Decreased circulating mtDNA levels in professional male volleyball players. Int $\mathrm{J}$ 261 Sports Physiol Perform. 11(1): 116-121. DOI: 10.1123/ijspp.2014-0461. PMID: 26226068407.

263 Nieman, D. C., Henson, D. A., McAnulty, S. R., McAnulty, L., Swick, N. S., Utter, A., 264 et al. 2002. Influence of vitamin C supplementation on oxidative and immune 265 changes after an ultramarathon. J App Physiol. 92(5): 1970-1977. DOI: 266 10.1152/japplphysiol.00961.2001. PMID: 11960947.

267 Nikolaidis, M. G., Kyparos, A., Spanou, C., Paschalis, V., Theodorou, A. A., \& 268 Vrabas, I. S. 2012. Redox biology of exercise: an integrative and comparative con269 sideration of some overlooked issues. J Exp Biol. 215(Pt 10): 1615-1625. DOI: 270 10.1242/jeb.067470. PMID: 22539728.

271 Twist, C., \& Highton, J. 2013. Monitoring fatigue and recovery in rugby league 272 players. Int J Sports Physiol Perform. 8(5): 467-474. PMID: 23319463.

273 Yamazaki, T., Kawai, C., Yamauchi, A., \& Kuribayashi, F. 2011. A highly sensitive 274 chemiluminescence assay for superoxide detection and chronic granulomatous 275 disease diagnosis. Trop Med Health. 39(2): 41-45. DOI: 10.2149/tmh.2011-08. 276 PMID: 22028609. 
Table 1. Physical characteristics, training history and marathon performance for the beetroot juice (BTJ) and placebo (PLA) groups.

\begin{tabular}{lcc}
\hline & PLA (n= 7) & BTJ (n=7) \\
\hline Age (years) & $45 \pm 10(27-60)$ & $45 \pm 7(36-60)$ \\
Sex (M/F) & $10 / 7$ & $11 / 6$ \\
Height (m) & $1.71 \pm 0.09(1.58-1.79)$ & $1.72 \pm 0.08(1.60-1.82)$ \\
Mass (kg) & $69.7 \pm 10.4(55.4-84.2)$ & $71.0 \pm 11.3(60.9-77.4)$ \\
No. of years running & $13 \pm 11(2-35)$ & $10 \pm 9(2-25)$ \\
Training distance (miles/week) & $38 \pm 14(20-55)$ & $41 \pm 16(18-65)$ \\
Longest training run (miles) & $22 \pm 3(18-26)$ & $25 \pm 6(20-38)$ \\
Previous marathons completed & $15 \pm 15(5-49)$ & $40 \pm 61(1-168)$ \\
Personal best time (hh:min) & $03: 47 \pm 0.02(03: 04-04: 47)$ & $03: 49 \pm 0.03(02: 59-04: 55)$ \\
Predicted finish time (hh:min) & $04: 17 \pm 0.02(03: 45-05: 00)$ & $04: 26 \pm 0.03(03: 45-05: 30)$ \\
Actual finish time (hh:min) & $04: 39 \pm 0.03(03: 59-05: 27)$ & $04: 43 \pm 0.03(03: 49-05: 38)$ \\
Pre-post change in body mass (kg) & $1.4 \pm 0.9(-0.5--2.7)$ & $1.3 \pm 1.0(+0.7--2.2)$. \\
Pre-post plasma volume change & $-18.6 \pm 20.1(-51.8-+5.5)$ & $-13.9 \pm 10.9(-24.2-+4.1)$ \\
\hline
\end{tabular}


Figure 1. Reactive oxygen species in the beetroot juice (BTJ) and placebo (PLA) groups before and after the marathon. "Different from pre-supplementation and preexercise $(P<0.05)$. Boxplots show mean \pm SD and $25-75^{\text {th }}$ percentiles with Tukeys rule for outliers $(\cdot)$.

Figure 2. mtDNA damage in the beetroot juice (BTJ) and placebo (PLA) groups before and after the marathon. Boxplots show mean \pm SD and $25-75$ th percentiles. 


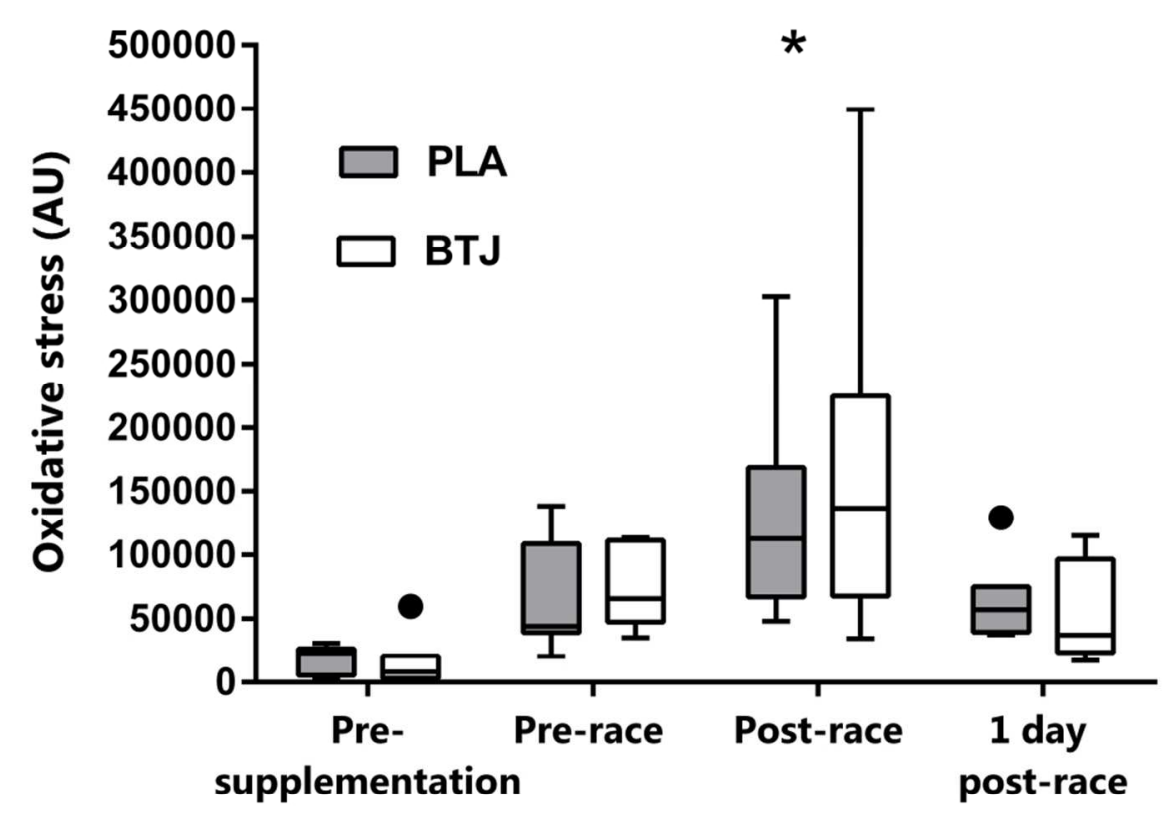

Time

Figure 1. Reactive oxygen species production in the beetroot juice (BTJ) and placebo (PLA) groups before and after the marathon. *Different from pre-supplementation and pre-exercise $(P<0.05)$. Boxplots show mean \pm SD and $25-75$ th percentiles with Tukeys rule for outliers $(\bullet)$.

$95 \times 70 \mathrm{~mm}(300 \times 300 \mathrm{DPI})$ 


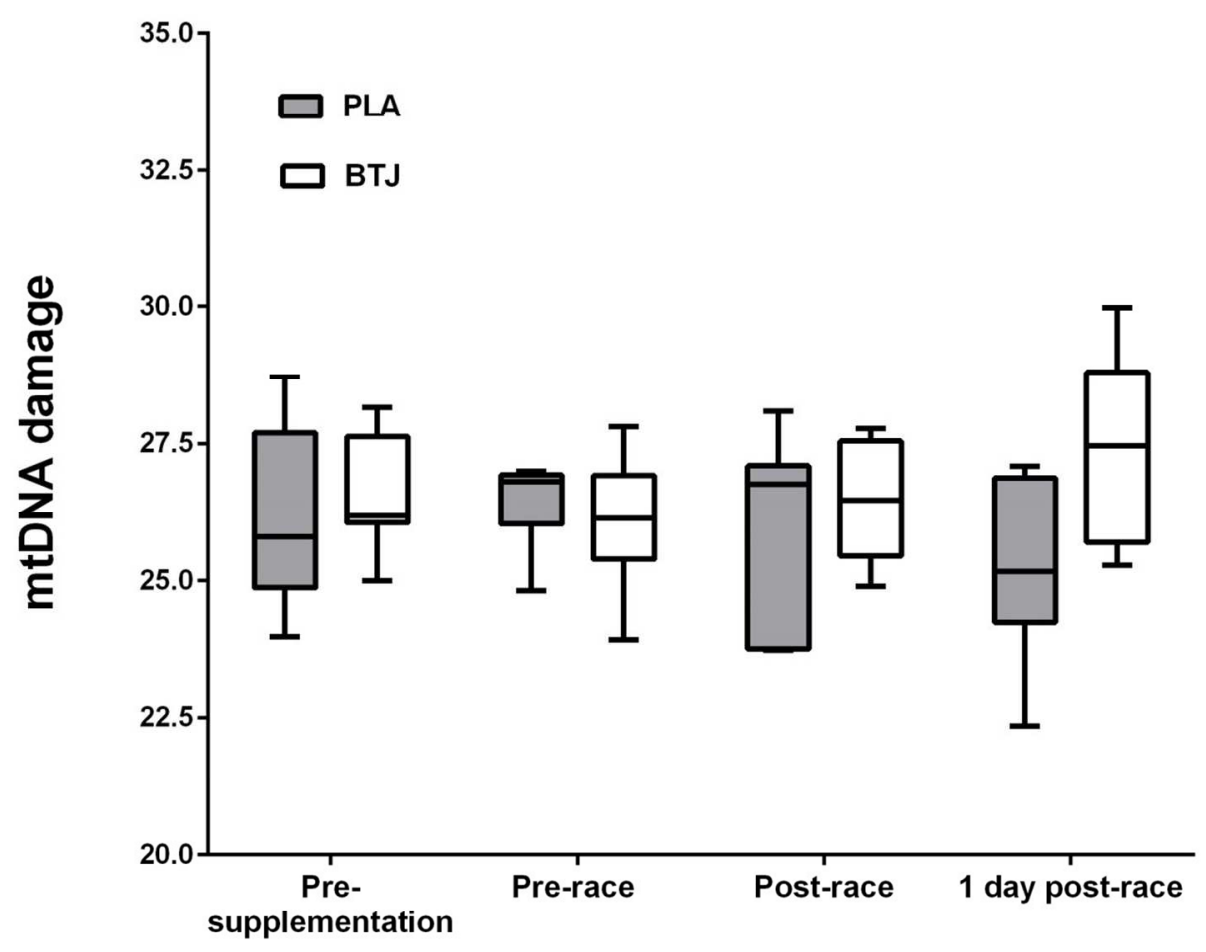

Time

Figure 2. mtDNA damage in the beetroot juice (BTJ) and placebo (PLA) groups before and after the marathon. Boxplots show mean \pm SD and $25-75$ th percentiles.

$$
116 \times 95 \mathrm{~mm}(300 \times 300 \mathrm{DPI})
$$

